\title{
Anterolateral Cervical Kyphoplasty for Metastatic Cervical Spine Lesions
}

\author{
Amer Sebaaly ${ }^{1,2}$, Ahmed Najjar ${ }^{3}$, Zhi Wang ${ }^{1}$, Ghassan Boubez ${ }^{1}$, Laura Masucci ${ }^{4}$, Daniel Shedid ${ }^{3}$ \\ ${ }^{1}$ Department of Orthopedic Surgery, Spine Unit, Centre Hopitalier de l'Université de Montréal, Montréal, QC, Canada \\ ${ }^{2}$ Faculty of Medicine, Saint Joseph University, Beirut, Lebanon \\ ${ }^{3}$ Department of Neurosurgery, Spine Unit, Centre Hopitalier de l'Université de Montréal, Montréal, QC, Canada \\ ${ }^{4}$ Department of Radiation Oncology, Spine Unit, Centre Hopitalier de l'Université de Montréal, Montréal, QC, Canada
}

\begin{abstract}
Study Design: Retrospective case series.
Purpose: To evaluate the clinical and radiological efficacy of anterolateral kyphoplasty for cervical spinal metastasis.

Overview of Literature: Although the spine is the third most common site of tumor metastasis, the cervical spine is the least commonly affected (incidence, 10\%-15\%). Surgical decompression is highly challenging because of the proximity of neural and vascular elements. Kyphoplasty for cervical spine metastasis has been described in small case reports with promising results.

Methods: Retrospective analysis of a prospective collected single-center spine metastasis database was done for cervical kyphoplasty cases. Data pertaining to age, sex, primary tumor diagnosis, modified Tokuhashi score, Spinal Instability Neoplastic Score (SINS), preoperative Visual Analog Scale (VAS) score, and analgesic medication were extracted. Postoperative data included VAS score at postoperative day 1, duration of hospitalization, self-reported functional outcome, and VAS score at the last follow-up.

Results: Eleven patients (mean age, 62.5 years) with cervical spine metastases were treated with 15-level kyphoplasty. Mean Tokuhashi score was 8.1, and mean SINS was 7.85. Mean preoperative pain score was 7.1, and 82\% of patients used opioid analgesics. Mean total bleeding volume was $100 \mathrm{~mL}$. Mean complication-free length of stay was 2.6 days with a decrease in postoperative pain (VAS score $=2.8, p<0.05)$. There was a $56 \%$ decrease in opioid dosage and the number of consumed analgesics $(1.09, p=0.004)$. Eightytwo percent of the patients reported excellent improvement at the last follow-up self-assessment.

Conclusions: To our knowledge, this case series represents the largest series of vertebral augmentation using balloon kyphoplasty for cervical spinal metastasis. This technique is associated with low postoperative complications as well as significant decrease in pain, use of opioids, and length of hospital stay. The main indications for vertebral kyphoplasty are lytic lesions of the cervical spine, painful lesions refractory to medical treatment, SINS score of 6-10, and absence of posterior wall defect.
\end{abstract}

Keywords: Cervical vertebrae; Spine; Neoplasm metastasis; Kyphoplasty; Palliative care

\section{Introduction}

The spine is the third most common site of metastasis after the lungs and the liver and is the most common site in the osteoarticular system [1]. Only a third of all patients with spinal metastasis are symptomatic and require

Received Sep 28, 2017; Revised Feb 6, 2018; Accepted Mar 19, 2018

Corresponding author: Amer Sebaaly

Department of Orthopedic Surgery, Spine Unit, Centre Hopitalier de l’Université de Montréal, Hôpital Notre Dame, Rue Sherbrooke, Montréal, QC, H2L 4M1, Canada

Faculty of Medicine, Saint Joseph University, Beirut, Lebanon

Tel: +961-3-679-128, E-mail: amersebaaly@hotmail.com 
medical or surgical intervention $[2,3]$. When the spine is involved (10\% of all tumor cases), the cervical spine is the least commonly affected $(10 \%-15 \%$ of all spinal metastasis) [3,4]. Surgical intervention is indicated in patients with neurological involvement, radioresistant tumor, or spinal instability and rarely for tissue diagnosis [5-7]. Surgical techniques aim to decompress neural structures, restore spinal stability, and alleviate pain.

Cervical spine metastasis is typically treated with piecemeal decompression or palliative surgery because en bloc resection is associated with high morbidity and mortality [4]. Moreover, surgical decompression is highly challenging because of the proximity of neural and vascular elements as well as because of the need for anterior and posterior approaches due to the highly mobile nature of this spinal segment [8]. Anterior cervical corpectomy offers the most direct approach in a majority of patients with cervical spine metastases. It allows for the decompression of the neural elements, excision of tumor, and effective reconstruction of the vertebral column [9]. Nonetheless, this approach is associated with a wide range of complications (reported incidence range, 4\%-66\%) [9].

Vertebroplasty was first described as a treatment option for aggressive hemangiomas of the lumbar spine [10]. The most common indication for vertebroplasty is vertebral osteoporotic fractures [11]. This treatment has been shown to be effective for the palliative treatment of metastatic spinal disease and even for the treatment of osteoblastic lesions [12]. Recent reports have shown promising results of kyphoplasty for cervical spine metastasis $[13,14]$. The objective of this study was to report the single-center experience with the palliative treatment of cervical metastasis using cervical kyphoplasty.

\section{Materials and Methods}

This study was approved by the Institutional Ethical Committee of the Centre Hopitalier de l'Université de Montréal, Montréal (IRB approval no., 17.156) and informed consent was waived. A prospectively collected database of a tertiary referral spine center was reviewed for cases of cervical spinal metastasis treated between January 2010 and the day of preparation of the manuscript. All patients had undergone magnetic resonance imaging (MRI) and computed tomography (CT) of the cervical spine to identify posterior cortical breach. Inclusion criteria were as follows: (1) age $>18$ years; (2) metastasis to the cervical spine; and (3) axial pain (Visual Analog Scale [VAS] score $\geq 6 / 10$ ). Exclusion criteria were patients who presented with neurological involvement and patients with highly unstable metastatic lesions (Spinal Instability Neoplastic Score [SINS] $\geq 12$ ) [7].

\section{Surgical technique}

The surgical technique for kyphoplasty for the cervical spine was described by Fuentes et al. [14] in 2009. With the patient in dorsal decubitus position and under general anesthesia, a right anterolateral approach at the approximate level of the cricoid cartilage was undertaken, keeping in mind the orientation of the odontoid for $\mathrm{C} 2$ kyphoplasty (exactly like a C2 odontoid screw placed in fractures). Generally, this incision may be used to treat multiple levels. The spine was dissected. Platysma dissection was performed and the medial border of the sternocleidomastoid muscle was localized. Next, the avascular plane is followed and the prevertebral fascia is opened. Special care should be taken during dissection of the pharyngeal fascia at the level of $\mathrm{C} 2-\mathrm{C} 3$. We recommend the use of the Synframe retractor system (Synthes, Paoli, PA, USA) for the retraction of the vital structures. Using fluoroscopy (AP and lateral), the Jamshidi needle was advanced at the lower anterior corner at the midline of $\mathrm{C} 2$ at the $\mathrm{C} 2-\mathrm{C} 3$ junction. The needle should have a trajectory that aims at the posterosuperior angle of the dens (similar to an odontoid screw). Next, the needle was advanced into the vertebral body and replaced with a working canula (10F canula) using a guidewire. Subsequently, we introduced and inflated the balloon under real-time fluoroscopic guidance. We recommend the use of smaller balloon in the cervical spine than that used in the thoracic and lumbar spine (generally $10 \mathrm{~mL}$ balloon, i.e., the smallest balloon for thoracic vertebra). Finally, the cement was prepared and injected under fluoroscopic guidance after deflation and removal of the balloon (Fig. 1). To prevent cement leakage, meticulous care was exercised by rigorous fluoroscopy control (approximately $2-3 \mathrm{~mL}$ per vertebra).

\section{Methods}

Background data included age, sex, and primary tumor diagnosis. Modified Tokuhashi score, SINS for the affected vertebra, and the number of vertebrae involved were also recorded. Patient characteristics included pre- 

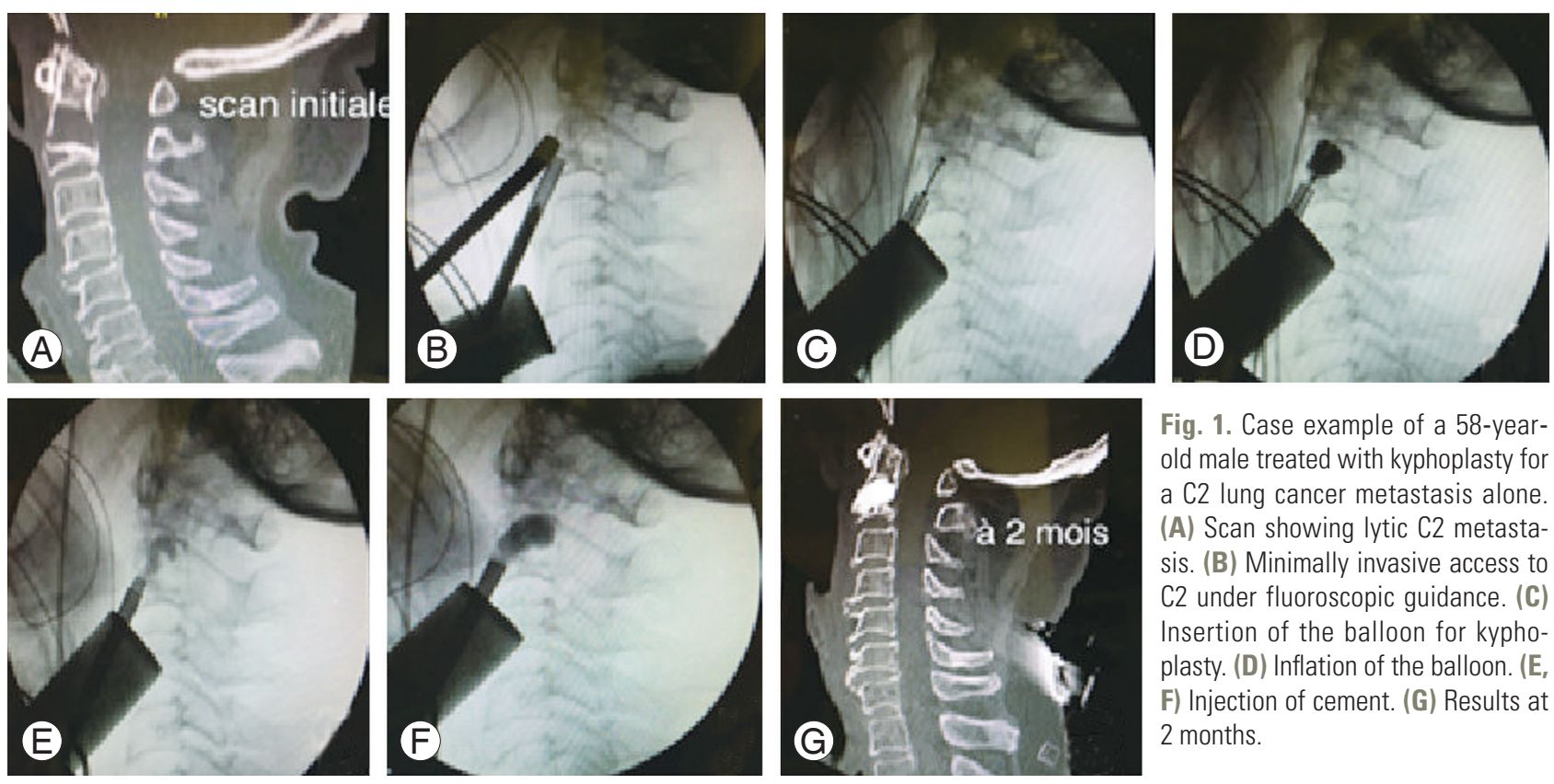

Fig. 1. Case example of a 58-yearold male treated with kyphoplasty for a C2 lung cancer metastasis alone. (A) Scan showing lytic C2 metastasis. (B) Minimally invasive access to C2 under fluoroscopic guidance. (C) Insertion of the balloon for kyphoplasty. (D) Inflation of the balloon. (E, F) Injection of cement. (G) Results at 2 months.

operative VAS score as well as opioid use. Postoperative data included VAS score at postoperative day 1, duration of hospitalization, self-reported functional outcomes (satisfaction with surgical outcomes and self-valuation of the outcome), VAS score at the last follow-up, and duration of follow-up.

Statistical analysis was performed using the PASW SPSS for Windows ver. 18.0 (SPSS Inc., Chicago, IL, USA). Student $t$-test was used to compare mean values, and chisquare test was used to compare binary variables. All $p<0.05$ were considered statistically significant.

\section{Results}

Overall, 11 patients (seven females and four males) with cervical spine metastases treated with 15-level kyphoplasty and vertebral body augmentation vertebroplasty were included (Table 1). Mean age of patients was 62.5 years.

Mean number of comorbid conditions was 1.45 (standard deviation $[\mathrm{SD}]=1.2$ ), mean Tokuhashi score was 8.1 $(\mathrm{SD}=2.3)$, mean SINS score was $7.85(\mathrm{SD}=1.1)$, and mean preoperative pain score was $7.1(\mathrm{SD}=1.7)$. Mean number of preoperatively consumed analgesics was $1.8(\mathrm{SD}=0.75)$; $82 \%$ of patients were using opioids, most commonly morphine or hydromorphone. Mean number of affected levels was $1.72(\mathrm{SD}=1.1)$. Seven patients $(63.6 \%)$ had single-level disease, whereas four had more than one cervical vertebrae affected. C2 was the most commonly affected level
(45\% of the operated cases). One patient required fusion for C4 instability in addition to kyphoplasty (SINS=11), and the remaining patients underwent kyphoplasty alone (91\%). The most common primary tumor was lung cancer (36\%), followed by multiple myeloma and breast cancer (each, 27\%).

Mean operative time was 75 minutes (including anesthesia time, $S D=46.7$ ), and mean total bleeding volume was $100 \mathrm{~mL}$ (SD=64.5). Mean number of operated levels was 1.45 . Two patients (18\%) developed major complications. One patient developed pulmonary embolism, which resulted in prolonged hospitalization. The other patient exhibited recurrent episodes of desaturation postoperatively (past medical history of emphysema), which led to respiratory decompensation and death on postoperative day 6. Mean complication-free length of stay was 3.9 days $(\mathrm{SD}=3.1)$. Pain in the postoperative period (mean VAS score $=2.8, \mathrm{SD}=1.47$ ) was significantly lower than that in the preoperative period $(p<0.001)$. Mean number of postoperatively consumed analgesics was significantly reduced to 1.09 ( $\mathrm{SD}=0.7, p=0.004)$. There was a $56 \%$ decrease in opioid dosage postoperatively. There was cement leakage in two levels (first cases were we would inject fresh prepared cement) with an incidence of $6 \%$.

Mean duration of follow-up was 6.1 months. Pain reduction was preserved at the last follow-up (VAS score $=3.1, p>0.05$ versus immediate postoperative VAS score). Moreover, $82 \%$ of the patients reported excellent 

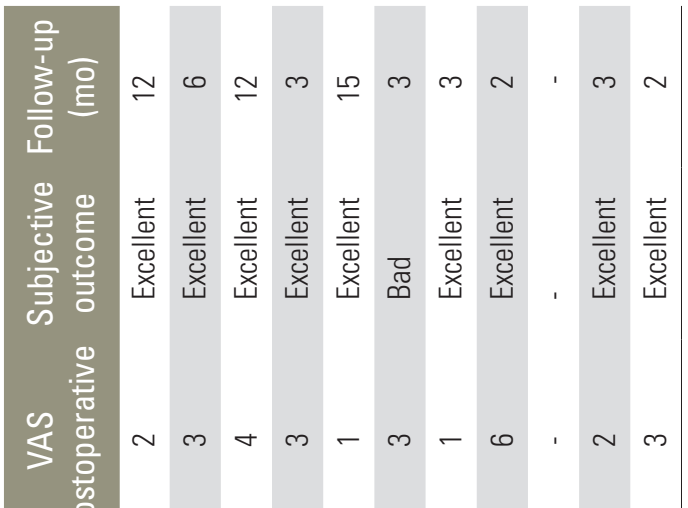

言

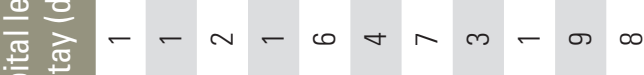

온

क्ष.

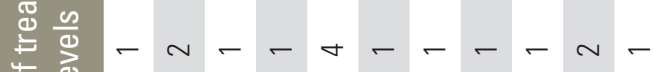

ì

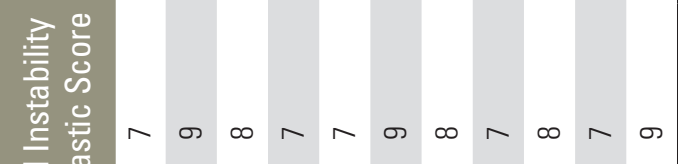

元 $\frac{0}{\frac{0}{0}}$

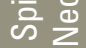

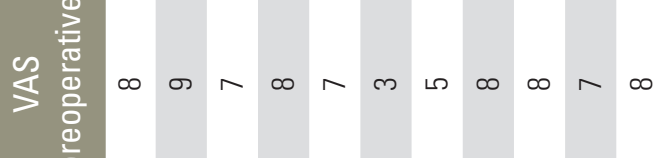

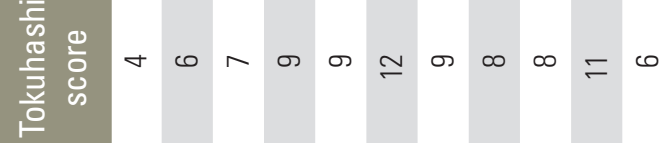

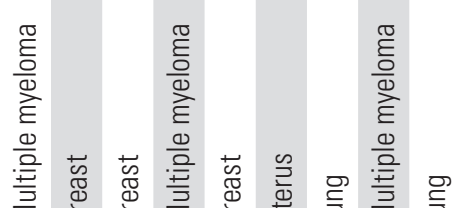

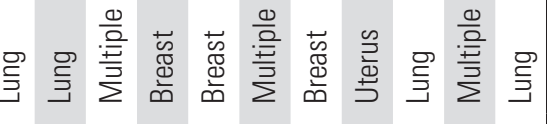

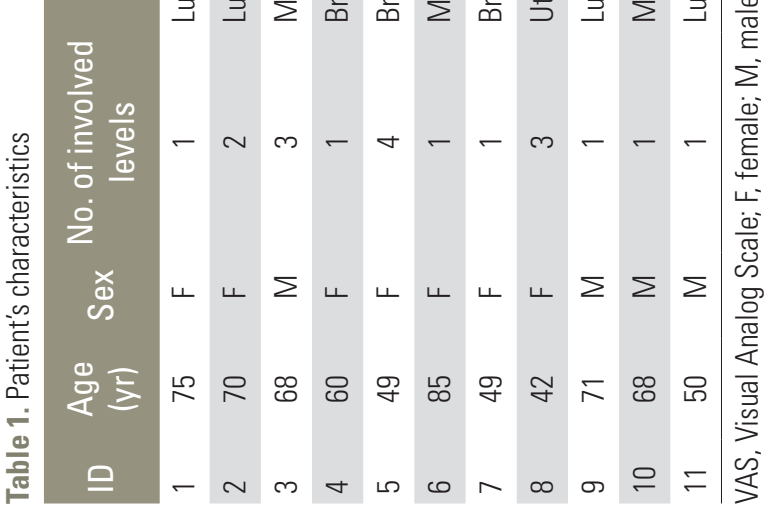

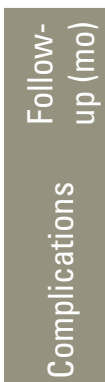

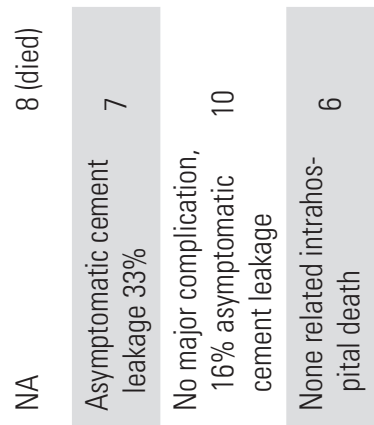

离

$\stackrel{\infty}{>}$

흘

$\frac{0}{8}$

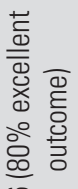

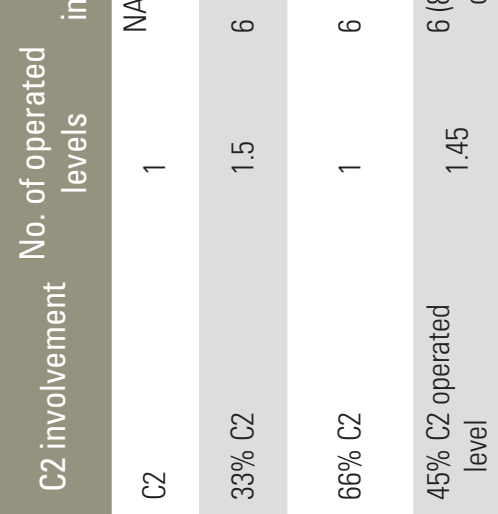

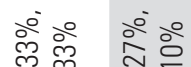

$\stackrel{2}{\gtrless}$

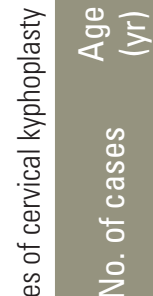

芯㐫

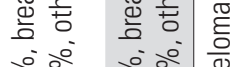

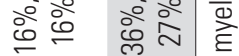

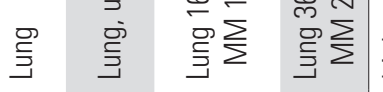

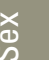

$\widetilde{0}$

오ํ

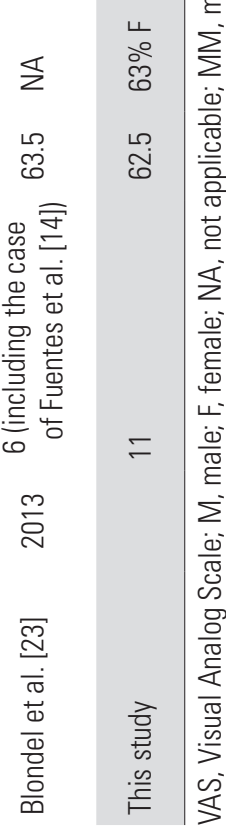


improvement on the last follow-up self-assessment. Only one patient developed local progression of the disease; however, reoperation was not performed because of poor overall health status.

\section{Discussion}

Spinal column metastasis is a common pathology. Postmortem pathological examination shows occult spinal metastasis in $30 \%-90 \%$ of patients with tumors, depending on the primary tumor type [15]. Although these metastases affect the cervical spine in only $8 \%-15 \%$ of cases, these patients often experience significant pain and discomfort because majority of the metastasis in the $\mathrm{C} 1-$ $\mathrm{C} 2$ area present with a fracture [8]. Pain in cancer patients is multifactorial and may be caused by pathologic compression fractures, invasion of paravertebral spinal tissues, and/or compression of neural elements [16]. In advanced stages, these patients have reduced life expectancy, with the strongest predictor of survival being the primary tumor type. Radiotherapy is one of the most commonly used palliative treatments for painful metastasis. Nonetheless, it is only effective in approximately $50 \%$ of patients with bone pain [16]. Classically, anterior corpectomy and fusion or posterior decompression and fusion have been the traditional method for the treatment of cervical spine metastasis [9]. Nonetheless, it is associated with high rates of complications; in particular, infection is a frequent occurrence in these patients [9]. Cement augmentation techniques represent a novel therapeutic approach that aims to decrease pain, stabilize spine, improve the quality of life, and avoid complex surgeries and their complications [12]. To date, this study represents the largest series of kyphoplasty for the treatment of cervical spine metastasis. Excellent results may be expected in more than $80 \%$ of the patients, with very low surgical complication rate related to the surgical technique. Another important finding is the possibility of a safe multilevel treatment using the same anterolateral approach.

Cement augmentation has been shown to improve the quality of life and decrease the consumption of analgesics at 1 year. Indeed, it is a well-established treatment for thoracolumbar spinal metastases [17]. The main indication for cement augmentation is the presence of mechanical pain (with/without vertebral compression fractures) with no evidence of neural compression. Nonetheless, the use of cement augmentation for cervical metastasis is not well reported because the typical characteristics of cervical spine (proximity to neural and vascular elements and small pedicles) render the procedure challenging [18]. Cement augmentation for cervical spinal metastasis is not widely studied; only 12 studies have reported cement augmentation (combined $n=113$ ) $[18,19]$. Pooled analysis of these results showed decrease in pain, improvement in quality of life, and decrease in the use of cervical collar. Nonetheless, vertebroplasty is associated with the injection of less viscose cement with the possibility of extravasation into the surrounding tissues, particularly if there is a breach of the vertebral posterior $[19,20]$. Stangenberg et al. [19] reported a 7.4\% rate of posterior cement extravasation, which may lead to serious consequences, as highlighted by Mont'Alverne et al. [21].

Balloon kyphoplasty creates a space for cement filling by decreasing the filling pressure, and allows injection of more viscous cement and decreases paravertebral and intradiscal cement extravasation [11]. Balloon kyphoplasty for cervical spinal metastasis was described by Fuentes et al. [14] in 2009; however, there are few reports of surgical efficacy of this technique (Table 2) [22-24]. To our knowledge, this study reports the largest series in the literature with excellent results in more than $80 \%$ of the patients, mean decrease in neck pain score by 5 points, and more than $50 \%$ reduction in pain medication and opioid use. Lykomitros et al. [22] presented a case series of two cases with two level kyphoplasty in one patient. They reported improvement in neck pain by 6 points on the VAS scale. Both patients did not require cervical collar postoperatively owing to subsidence of neck pain and stiffness [22]. The second largest series was reported by Blondel et al. [23] Their findings are consistent with the findings of the present study: improvement of 6 points on the VAS score, low bleeding volumes ( $<200 \mathrm{~mL}$ in all cases), and length of stay of 2 days [23]. Nonetheless, there were no cases of multiple level application of this technique. Posterior wall defect remains the only contraindication to this technique, which calls for detailed evaluation of each patient with MRI and CT.

First of all, the included number of patients is too low to allow for robust statistical analysis including correlation analysis. However, the observed statistical difference could be interpreted with strong power since the difference is highly significant and represents the largest series in the literature. Another limitation of this study is the short duration of follow-up. Unfortunately, our institution 
is a tertiary referral center, and patients tend to continue follow-ups in their respective hospitals in far areas in the province.

\section{Conclusions}

As per our knowledge, this study represents the largest series of patients with cervical spinal metastasis who underwent vertebral augmentation using balloon kyphoplasty. This technique is associated with low postoperative complications as well as significant decrease in pain, use of opioids, and length of hospital stay. We would like to emphasize on the feasibility of the use of the same approach at multiple levels with no increase in surgical bleeding or complications. We believe that the main indications for vertebral kyphoplasty are lytic lesions of the cervical spine, painful lesions that are refractory to medical treatment, SINS score of 6-10, and absence of posterior wall defect.

\section{Conflict of Interest}

No potential conflict of interest relevant to this article was reported.

\section{References}

1. Quraishi NA, Rajabian A, Spencer A, et al. Reoperation rates in the surgical treatment of spinal metastases. Spine J 2015;15(3 Suppl):S37-43.

2. Ibrahim A, Crockard A, Antonietti P, et al. Does spinal surgery improve the quality of life for those with extradural (spinal) osseous metastases?: an international multicenter prospective observational study of 223 patients: invited submission from the Joint Section Meeting on Disorders of the Spine and Peripheral Nerves, March 2007. J Neurosurg Spine 2008;8:271-8.

3. Sciubba DM, Gokaslan ZL, Suk I, et al. Positive and negative prognostic variables for patients undergoing spine surgery for metastatic breast disease. Eur Spine J 2007;16:1659-67.

4. Cho W, Chang UK. Neurological and survival outcomes after surgical management of subaxial cervical spine metastases. Spine (Phila Pa 1976) 2012;37:E969-77.

5. Dodwad SN, Savage J, Scharschmidt TJ, Patel A.
Evaluation and treatment of spinal metastatic disease. Cancer Treat Res 2014;162:131-50.

6. Patchell RA, Tibbs PA, Regine WF, et al. Direct decompressive surgical resection in the treatment of spinal cord compression caused by metastatic cancer: a randomised trial. Lancet 2005;366:643-8.

7. Fisher CG, DiPaola CP, Ryken TC, et al. A novel classification system for spinal instability in neoplastic disease: an evidence-based approach and expert consensus from the Spine Oncology Study Group. Spine (Phila Pa 1976) 2010;35:E1221-9.

8. Molina CA, Gokaslan ZL, Sciubba DM. Diagnosis and management of metastatic cervical spine tumors. Orthop Clin North Am 2012;43:75-87.

9. Sayama CM, Schmidt MH, Bisson EF. Cervical spine metastases: techniques for anterior reconstruction and stabilization. Neurosurg Rev 2012;35:463-74.

10. Galibert P, Deramond H, Rosat P, Le Gars D. Preliminary note on the treatment of vertebral angioma by percutaneous acrylic vertebroplasty. Neurochirurgie 1987;33:166-8.

11. Sebaaly A, Nabhane L, Issa El Khoury F, Kreichati G, El Rachkidi R. Vertebral augmentation: state of the art. Asian Spine J 2016;10:370-6.

12. Tian QH, Sun XQ, Lu YY, et al. Percutaneous vertebroplasty for palliative treatment of painful osteoblastic spinal metastases: a single-center experience. J Vasc Interv Radiol 2016;27:1420-4.

13. Blondel B, Adetchessi T, Demakakos J, Pech-Gourg G, Dufour H, Fuentes S. Anterolateral kyphoplasty in the management of cervical spinal metastasis. Orthop Traumatol Surg Res 2012;98:341-5.

14. Fuentes S, Metellus P, Pech-Gourg G, Dufour H, Grisoli F. Surgical kyphoplasty for management of axis metastasis: technical note kyphoplastie de C2. Neurochirurgie 2009;55:323-7.

15. Wong DA, Fornasier VL, MacNab I. Spinal metastases: the obvious, the occult, and the impostors. Spine (Phila Pa 1976) 1990;15:1-4.

16. Meeuse JJ, van der Linden YM, van Tienhoven G, et al. Efficacy of radiotherapy for painful bone metastases during the last 12 weeks of life: results from the Dutch Bone Metastasis Study. Cancer 2010;116:271625.

17. Anselmetti GC, Bonaldi G, Carpeggiani P, Manfre L, Masala S, Muto M. Vertebral augmentation: 7 years experience. Acta Neurochir Suppl 2011;108:147-61. 
18. Health Quality Ontario. Vertebral augmentation involving vertebroplasty or kyphoplasty for cancerrelated vertebral compression fractures: a systematic review. Ont Health Technol Assess Ser 2016;16:1-202.

19. Stangenberg M, Viezens L, Eicker SO, Mohme M, Mende KC, Dreimann M. Cervical vertebroplasty for osteolytic metastases as a minimally invasive therapeutic option in oncological surgery: outcome in 14 cases. Neurosurg Focus 2017;43:E3.

20. Sebaaly A, Rizkallah M, Bachour F, Atallah F, Moreau PE, Maalouf G. Percutaneous cement augmentation for osteoporotic vertebral fractures. EFORT Open Rev 2017;2:293-9.
21. Mont'Alverne F, Vallee JN, Cormier E, et al. Percutaneous vertebroplasty for metastatic involvement of the axis. AJNR Am J Neuroradiol 2005;26:1641-5.

22. Lykomitros V, Anagnostidis KS, Alzeer Z, Kapetanos GA. Percutaneous anterolateral balloon kyphoplasty for metastatic lytic lesions of the cervical spine. Eur Spine J 2010;19:1948-52.

23. Blondel B, Litre F, Graillon T, Adetchessi T, Dufour H, Fuentes S. Metastatic odontoid fracture management by anterior screw fixation and kyphoplasty. Neurochirurgie 2013;59:191-4.

24. Fransen P, Collignon FP. Direct anterolateral balloon kyphoplasty for a painful C-2 osteolytic malignant lesion. Case illustration. J Neurosurg Spine 2007;6:374. 